\title{
Binding global and local object features in visual working memory
}

\author{
Justin M. Ericson ${ }^{1,2} \cdot$ Melissa R. Beck ${ }^{1}$ • Amanda E. van Lamsweerde ${ }^{1,3}$
}

Published online: 28 October 2015

(C) The Psychonomic Society, Inc. 2015

\begin{abstract}
When briefly presented with global and local visual information, individuals report global information more quickly and more accurately than local information, a phenomenon known as the global precedence effect (GPE; Navon, 1977). We investigated whether a bias toward global information persists in visual working memory (VWM) and whether the VWM representations for global and local features include information bound to their hierarchical levels and to each other. Navon figures, in which a larger (global) letter is composed of smaller (local) letters, were presented, and participants performed a change detection task that required participants to remember features only (either a global or local letter changed to a new identity); features bound to their hierarchical levels (the global and local letters within an object swapped levels); or features bound to each other within an object ( 2 letters from the same level swapped between objects). Performance suggested that there was a GPE in VWM (new global letters were more accurately detected than new local letters) and that although global and local features were not necessarily bound together in VWM, they were bound to their corresponding hierarchical levels. These results indicate that level binding in VWM occurs more readily than binding specific object features together. These findings fur-
\end{abstract}

Justin M. Ericson

justin.ericson@duke.edu

1 Department of Psychology, Louisiana State University, Baton Rouge, LA, USA

2 Center for Cognitive Neuroscience, Duke University, Durham, NC, USA

3 Department of Psychology, North Dakota State University, Fargo, ND, USA ther our understanding of how hierarchical objects are represented in VWM.

Keywords Memory: visual working and short-term memory . Attention and memory $\cdot$ Visual working memory

Complex objects are often composed of multiple features (e.g., green, oval, vertical) at different hierarchical levels: global (e.g., a tree) and local (e.g., leaves or branches). Features of an object are initially processed independently of each other (Treisman \& Gelade, 1980) and their hierarchical levels (Hübner \& Volberg, 2005). However, perception of a unified object requires feature-to-feature ("object") binding (Treisman \& Gelade, 1980; Wheeler \& Treisman, 2002) and feature-to-level binding (Hübner \& Volberg, 2005). This binding process occurs via direction of spatial attention (Treisman \& Gelade, 1980). Furthermore, some bindings may occur more readily in perception than others, and the direction of attention can bias these effects (Boer \& Keuss, 1982; Flevaris, Bentin, \& Robertson, 2010; Miller, 1981). Although binding during perception has been studied extensively and is relatively well understood, less is known about the extent to which feature-to-feature and feature-to-level bindings of hierarchical objects are maintained in visual working memory (VWM).

\section{Binding in perception}

Individual features of an object are initially processed independently, after which they may be bound to their hierarchical levels and to each other, leading to the perception of a unified object. Navon (1977) demonstrated that global and local features are initially processed independently using stimuli (Navon figures) that consist of a large letter (global level) 
composed of smaller letters (local level). Letter identification was faster for the global level than for the local level, leading Navon to conclude that global features are processed prior to the local features. This effect is referred to as the global precedence effect (GPE).

Feature-to-level binding occurs after individual features have been identified (Hübner \& Volberg, 2005). When participants were tasked with reporting either the global level (large letter) or local level (small letter) of a Navon figure that was presented briefly $(24 \mathrm{~ms})$ and then masked at various intervals (12-96 ms), participants were more likely to make feature-tolevel errors (e.g., reporting the local letter when asked to report the global letter or vice versa) at short stimulus-to-mask intervals (Hübner \& Volberg). Therefore, at shorter intervals, participants had identified individual features, but had not yet created the appropriate feature-to-level binding; the featureto-level bindings were created only at the longer intervals. This suggests that feature-to-level bindings are perceived after feature identification.

This feature-to-level binding is dependent upon attention (Austen \& Enns, 2000; Flevaris et al., 2010; Lamb \& Yund, 1993, 1996). Priming attention toward high spatial frequency information, by asking participants to first identify the orientation of a high spatial frequency grating, can improve binding of features to the local level, and priming toward low spatial frequency information can improve binding of features to the global level (Flevaris et al., 2010). Similarly, according to feature integration theory, individual features (e.g., green and oval) can be perceived preattentively, but the feature-tofeature binding (green oval) occurs after spatial attention has been deployed to the object (Treisman \& Gelade, 1980). Although perceptually binding features to either their hierarchical levels or each other may be difficult when time constraints prevent the adequate deployment of attention, these biases may not persist into VWM provided there is adequate time and resources to encode information.

\section{Binding in memory}

The GPE has been found to continue into memory when there is a load on attention and VWM resources (Austen \& Enns, 2000; Rumiati, Nicoletti, \& Job, 1989); however, the maintenance of feature-to-level bindings in VWM has received little consideration. The authors are unaware of any previous research looking specifically at binding of hierarchical objects in memory. However, considerable research has been conducted on whether feature-to-feature bindings of an object are maintained in VWM.

Once an object is encoded into VWM, multiple features can be remembered as part of the object with no cost to performance (Luck \& Vogel, 1997). This would suggest that integrated objects are maintained in VWM, with all of the constituent features bound to each other. However, it can be more difficult to detect changes to object bindings (e.g., when a blue circle and a red square change to a blue square and a red circle) than to detect the presence of new features (e.g., a blue circle changes to a blue triangle; Wheeler \& Treisman, 2002). These binding changes are especially difficult to detect when the VWM test requires participants to shift attention away from memory representations (Wheeler \& Treisman). As a result, it has been suggested that sustained attention is required to maintain a bound representation in VWM; when attention is shifted away, individual features remain but feature bindings are lost. The question of whether feature bindings are maintained in VWM through sustained attention has been directly tested using various attention manipulations, but the results of these manipulations have been mixed (Allen, Baddeley, \& Hitch, 2006; Allen, Hitch, Mate, \& Baddeley, 2012; Brown \& Brockmole, 2010; Delvenne, Cleeremans, \& Laloyaux, 2010; Fougnie \& Marois, 2009; Gajewski \& Brockmole, 2006; Johnson, Hollingworth, \& Luck, 2008; Morey \& Bieler, 2013; van Lamsweerde \& Beck, 2012; Yeh, Yang, \& Chiu, 2005).

It is therefore an open issue whether integrated object representations are maintained in VWM. Furthermore, although prior investigation of this issue has been largely restricted to feature-to-feature bindings, many objects contain features at multiple levels. Therefore, the goal of the current study was to determine the extent to which object maintenance in VMW mirrors perception by investigating whether the GPE occurs in VWM, as well as whether feature-to-feature and feature-tolevel bindings of hierarchical objects are maintained in VWM.

\section{The current study}

An accurate VWM representation of a hierarchical object would contain the global and local features, the features bound to each other, and the features bound to their levels. To determine the extent to which all of these components are maintained in VWM representations, participants completed a change detection task in which several Navon figures were presented simultaneously in a study display, and at test a single stimulus probe was presented that was either the same as one of the studied objects or a modified version of one of the studied objects. This use of a single test object is typical in tests of feature binding memory because it prevents the use of location binding for completing the task (Johnson et al., 2008; Wheeler $\&$ Treisman, 2002). There were four possible modified versions of one of the studied Navons that could be presented at test: (1) the test object contained a studied global feature combined with a new local feature (new-local change); (2) the test object contained a studied local feature combined with a new global feature (new-global change); (3) the test object consisted of the studied global and local features of one of the studied objects, 
but the features swapped levels (feature-to-level binding change); or (4) the test object consisted of a local feature from one studied object and a global feature from different studied object (feature-to-feature binding change).

It was predicted that the GPE would be observed in VWM, as indicated by higher performance on the new-global trials than the new-local trials. Furthermore, because attention influences the GPE at the perceptual level (Flevaris et al., 2010), it was hypothesized that biasing attention toward the local level with a high spatial frequency prime would reduce the GPE in VWM, as evidenced by an increase in performance for new-local changes.

The critical test of whether features are bound to their levels in VWM was completed by comparing feature-to-level change detection rate to the highest single-feature change detection rate (in this case, the new-global changes). For example, if a participant studied an E made of Ss and was tested on an $\mathrm{S}$ made of Es, the feature-to-feature binding remains the same (i.e., E and $\mathrm{S}$ are still features of the same object), but the feature-to-level binding has changed. Accurate performance requires that at least one of the features (either the $\mathrm{E}$ or the $\mathrm{S}$ ) is encoded and bound to the correct level (either global or local, respectively); on any given trial, it is more likely that if only one feature of an object is encoded, it would be the feature with the highest change detection rate. Therefore, if feature-to-level bindings are represented in VWM, feature-tolevel changes should be detected as accurately as the newfeature trials (new-local or new-global) with the best performance; as predicted, these were the new-global trials.

When both the local and global features of an object are represented in VWM, it is possible that (1) the global and local features are maintained together as a bound representation or (2) both features are maintained, but independently and without the feature-to-feature bindings. To test between these hypotheses, feature-to-feature change detection (features of two different objects were swapped) was compared to the lowest single-feature change detection rate (new-local changes). Accurate performance on these trials requires that both features of an object are remembered, and that they are bound to each other (e.g., there was an $E$ and an $S$ that belong together), but it is not necessary to remember which feature was global and which was local. If the VWM representation is a unified object, participants should remember the feature-to-feature bindings only as long as they have encoded both individual features. Therefore, if feature-to-feature bindings are maintained in VWM, performance on feature-to-feature change trials should equal performance for detecting changes to the more difficult feature (Wheeler \& Treisman, 2002). In this study, new-local changes were more difficult to detect than new-global changes (as predicted); therefore, feature-tofeature performance was compared to new-local performance. A summary of the research questions investigated in this study is found in Table 1.

\section{Experiment 1}

\section{Method}

Participants Thirty-one (17 male and 14 female) undergraduate psychology students from Louisiana State University, with self-reported normal or corrected-to-normal vision and a mean age of 20.2 years ( $S D=1.31$ years) participated in the experiment for course credit.

Stimuli The experiment was administered with MATLAB utilizing the Psychophysics Toolbox extension (Brainard, 1997; Pelli, 1997) on four iMac 2 GHz Intel Core Duo computers. Participants sat unrestrained, approximately $57 \mathrm{~cm}$ from the display. Two types of stimuli were utilized: spatial frequency (SF) gratings to prime attention prior to the change detection task, and Navon figures, the stimuli to be remembered in the change detection task (see Fig. 1).

The high and low spatial frequency (SF) gratings were presented simultaneously at the center of the display, creating a compound grating with a diameter of approximately $12^{\circ}$ visual angle. Across the compound grating diameter, the low SF grating repeated its function at approximately $.83 \mathrm{c} / \mathrm{deg}$, whereas the high SF grating repeated its function at approximately $8.3 \mathrm{c} / \mathrm{deg}$. Finally, the high SF and low SF gratings were presented in opposing orientations of each other at either $+45^{\circ}$ (angled right) or $-45^{\circ}$ (angled left) from vertical (see Fig. 1).

Navon figures were composed of any possible large (global) and small (local) "digital" block letter (A, C, E, F, $\mathrm{H}, \mathrm{O}, \mathrm{P}, \mathrm{S}$, and $\mathrm{U}$ ) combination, except simultaneous combinations (e.g., a large $\mathrm{F}$ composed of small Fs), resulting in 72 large and small letter combinations. A letter could be used only once in a given study array. The small letters subtended approximately $.5^{\circ} \times 1^{\circ}$ of visual angle, while the large letters were assembled within of a $5 \times 5$ matrix of possible small letter locations resulting in a large letter that subtended approximately $3.5^{\circ} \times 6.5^{\circ}$ of visual angle. Navons were presented in black on a gray background. Masks were constructed to resemble number $8 \mathrm{~s}$ and were identical in size to the Navon figures. In each study display, three Navons were arranged around the center of the screen, such that the midpoint of each Navon was $5.23^{\circ}$ visual angle $\left(S D=.44^{\circ}\right)$ away from the center of the screen. Navons could appear in one of six locations (top right, top left, center left, bottom right, bottom left, and bottom) and were arranged on the screen in one of four configurations: (1) top left, top right, and bottom center; (2) bottom left, bottom right, and top center; (3) top left, bottom left, and right center; and (4) top right, bottom right, and left center.

Design A 3 (prime type) $\times 5$ (trial type) repeated measures design was employed. Prime type (no prime, global prime, 
Table 1 The four main research questions for Experiment 1 and the pattern of performance that would support or fail to support each question

\begin{tabular}{|c|c|c|}
\hline & Yes & No \\
\hline 1. GPE in VWM? & Higher new-global than new-local performance. & $\begin{array}{l}\text { Equivalent new-global and new-local } \\
\text { performance. }\end{array}$ \\
\hline 2. Does an attention prime affect the GPE in VWM? & $\begin{array}{l}\text { GPE in the no prime and global prime conditions, } \\
\text { but not in the local prime condition. }\end{array}$ & GPE in all prime conditions. \\
\hline 3. Feature-to-level binding in VWM? & $\begin{array}{l}\text { Equivalent feature-to-level and new-global } \\
\text { performance. }\end{array}$ & $\begin{array}{l}\text { Lower feature-to-level than new-global } \\
\text { performance. }\end{array}$ \\
\hline $\begin{array}{l}\text { 4. Feature-to-feature binding of hierarchical } \\
\text { features in VWM? }\end{array}$ & $\begin{array}{l}\text { Equivalent feature-to-feature and new-local } \\
\text { performance. }\end{array}$ & $\begin{array}{l}\text { Lower feature-to-feature than new-local } \\
\text { performance. }\end{array}$ \\
\hline
\end{tabular}

local prime) was manipulated within subjects and presented in blocks that were randomly ordered for each participant. Trial type was also manipulated within subjects with all trial types (no-change, new-global, new-local, feature-to-feature, and feature-to-level) randomly distributed within each prime type block.

Procedure The trial sequence for Experiment 1 can be seen in Fig. 1. Trials in the global prime and local prime blocks began with the presentation of a spatial frequency (SF) grating and instructions to respond to the tilt $\left(45^{\circ}\right.$ right or left from the vertical center) of either the high or low SF lines. This task primed attention to either global (low SF) or local (high SF) information (Flevaris et al., 2010). On half of these SF trials, participants reported the direction of tilt of the high SF (local prime trials), and on the other half of the trials, participants reported the direction of tilt of the low SF (global prime trials). Participants responded to a leftward tilt using the " 4 " key on the number pad and to a rightward tilt using the " 5 " key. Instructions were presented above the compound grating to aid the participant during this task. If the SF tilt was answered incorrectly, "INCORRECT, PLEASE TRY AGAIN" was presented on the screen in red for five seconds. Following an incorrect response, a new randomly chosen SF grating would be displayed. The trial would continue only after a correct response to the $\mathrm{SF}$ grating.

The change detection portion of the trial began with a $.5^{\circ}$ wide fixation target presented in the center of the display for $400 \mathrm{~ms}$, followed by the premasks for $500 \mathrm{~ms}$ in the same layout as the following Navon stimuli. After a $400 \mathrm{~ms}$ ISI, the three study Navon figures were presented for $500 \mathrm{~ms}$. After a $100 \mathrm{~ms}$ ISI, the postmasks were presented for $500 \mathrm{~ms}$. Finally, a single test Navon figure was presented in the center of the response screen. Participants determined whether the test figure was exactly the same (composed of the same local and global letters) as one of the figures presented on the study screen. Participants pressed either the " 1 " key, indicating that the test figure was present on the study screen, or the " 2 " key, indicating that the test figure was absent from the study screen.

The Navon figure on the response screen was either the same as one of the figures on the study screen (no-change),

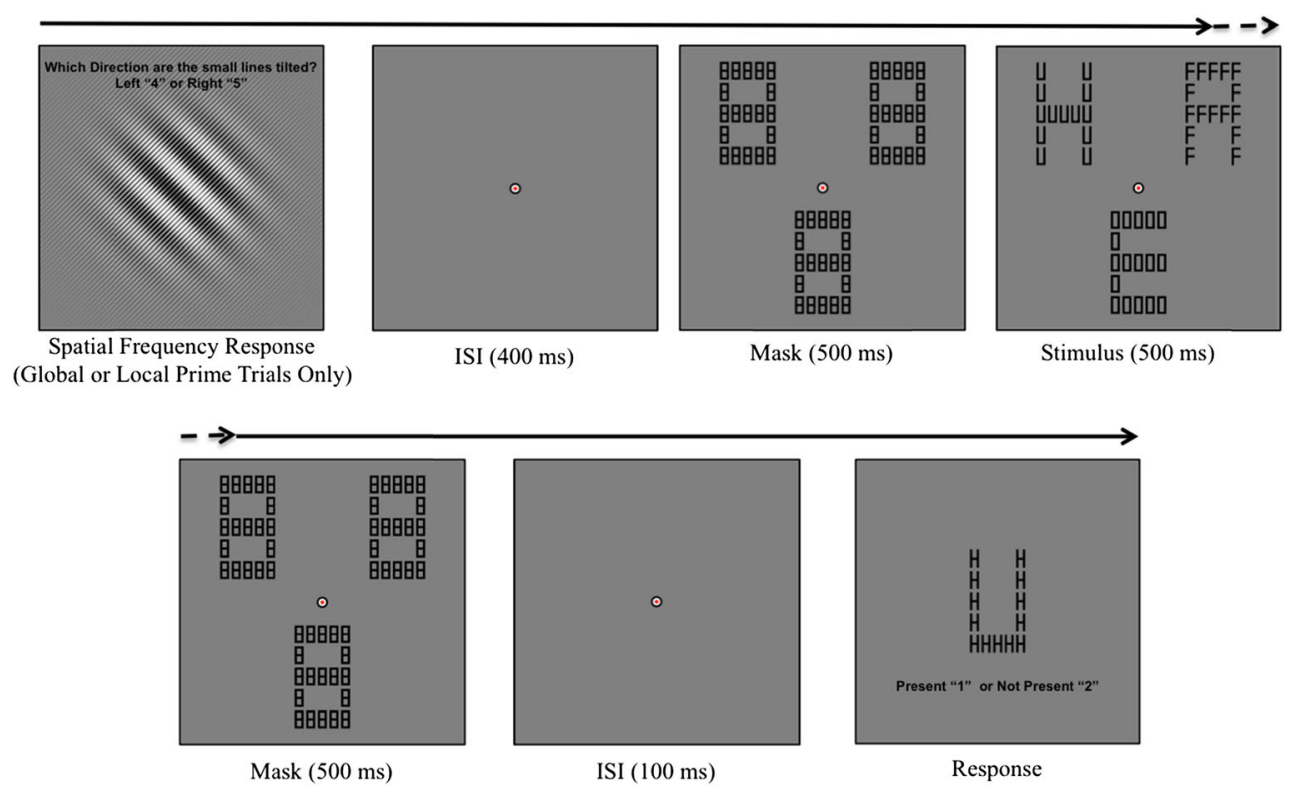

Fig. 1 Example stimulus sequence for Experiment 1 
or one of four types of changes occurred: (1) a new-global change, (2) a new-local change, (3) a feature-to-level change, or (4) a feature-to-feature change (see Fig. 2). A new-global change occurred when the test Navon figure was composed of a local feature of one of the study Navon figures and a global feature that was not presented at study. A new-local change occurred when a test Navon figure contained a global feature of one of the study Navon figures and a local feature that was not presented at study. A feature-to-level change occurred when the test figure was composed the same two letters as one of the study Navon figures, but the local feature (small letter) and global feature (large letter) swapped levels (e.g., a large E made of small Os at study and a large $\mathrm{O}$ made of small Es at test). A feature-to-feature change occurred when a local feature from one study Navon figure was combined with the global feature of another Navon (e.g., a large E made of small Os and a large A made of small Fs at study, and a large E made of small Fs at test).

Prior to the start of the experiment, participants were given both verbal and written instructions about how to perform the task. Participants completed six practice trials consisting of two trials without SF gratings (no prime), two trials requiring a response to low SFs (global prime), and two trials requiring a response to high SFs (local prime). All five trial types were randomized during the six practice trials. Following practice, participants completed three blocks (no prime, global prime, and local prime) of 96 trials each, 48 no-change, 12 new-global, 12 new-local, 12 feature-to-level, and 12 feature-to-feature, for a total of 288 experimental trials.

\section{Results}

The GPE, feature-to-level binding, and feature-to-feature binding effects were analyzed by comparing performance on the relevant change trials only. In all following analyses, proportion correct on the trials with a change (responding "not present") was used to calculate accuracy (see Fig. 3).

Global precedence effect To determine whether a GPE occurs in VWM, and whether the attention prime affected the GPE, the new-global and new-local trials were compared across all three attention primes (no prime, global prime, local prime) with a 2 (trial type) $\times 3$ (attention prime) repeated measures ANOVA (the patterned bars in Fig. 3). This analysis revealed a GPE, as overall performance was higher on the new-global trials $(M=.71, S E=.03)$ than on the new-local trials $(M=.59, S D=.03), F(1,30)=8.55, p=.007, \eta_{\mathrm{p}}{ }^{2}=.22$. However, there was no effect of attention prime, $F(2,60)=$ $.413, p=.663, \eta_{\mathrm{p}}{ }^{2}=.01$, and no interaction between trial type and attention prime, $F(2,60)=2.50, p=.090, \eta_{\mathrm{p}}{ }^{2}=.08$.

Feature-to-level binding To investigate whether features are bound to their hierarchical level and whether this is

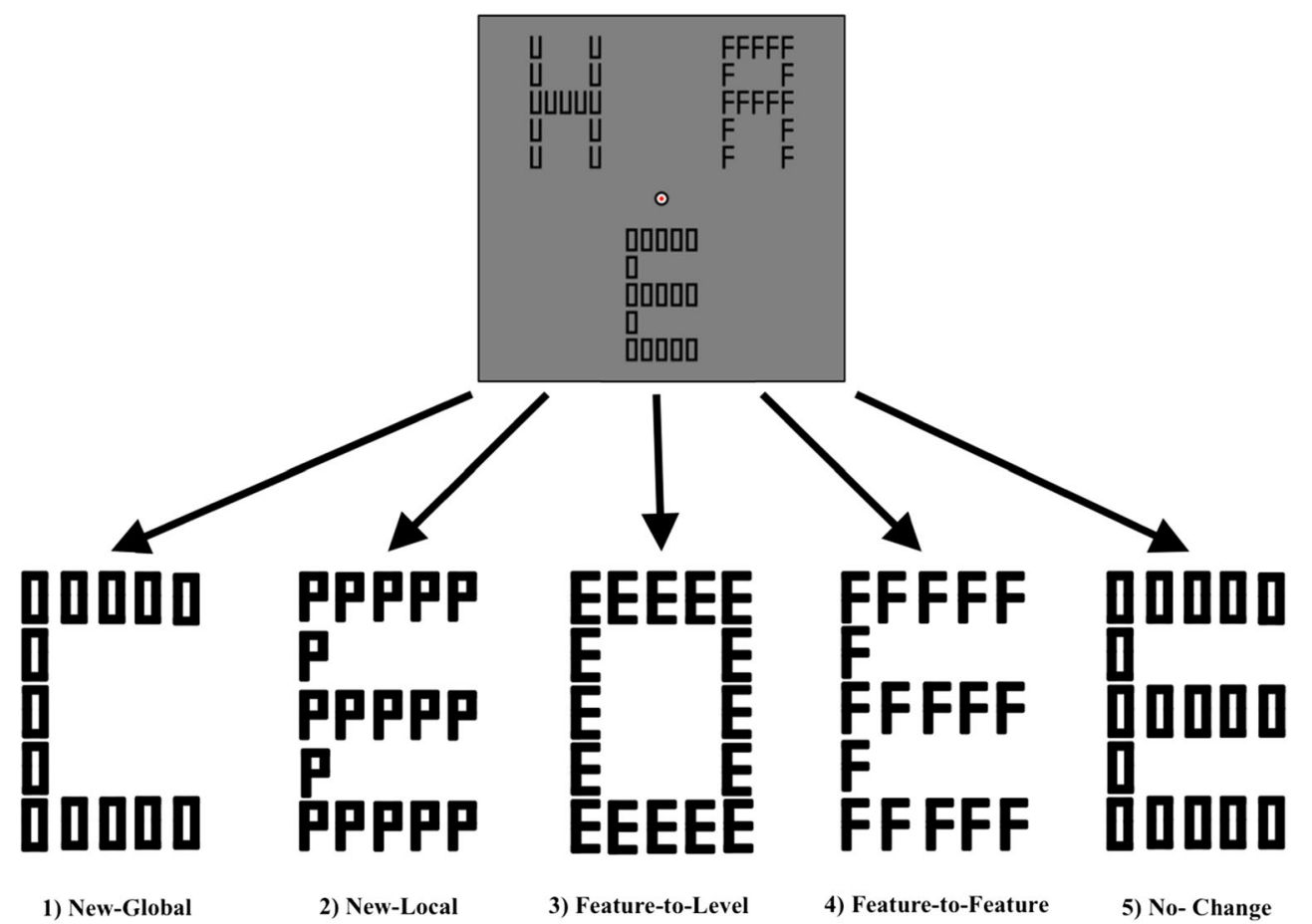

Fig. 2 Schematic representing each change type for a Navon constructed with a global (large letter) E made of local (small letters) Os: (1) newglobal change - global C made of local Os; (2) new-local change - a global E made of local Ps; (3) feature-to-level change - global O made of

local Es; (4) feature-to-feature change - global E made of local Fs (note that another item in the study array had local Fs); and (5) no-change - a global E made of local Os 


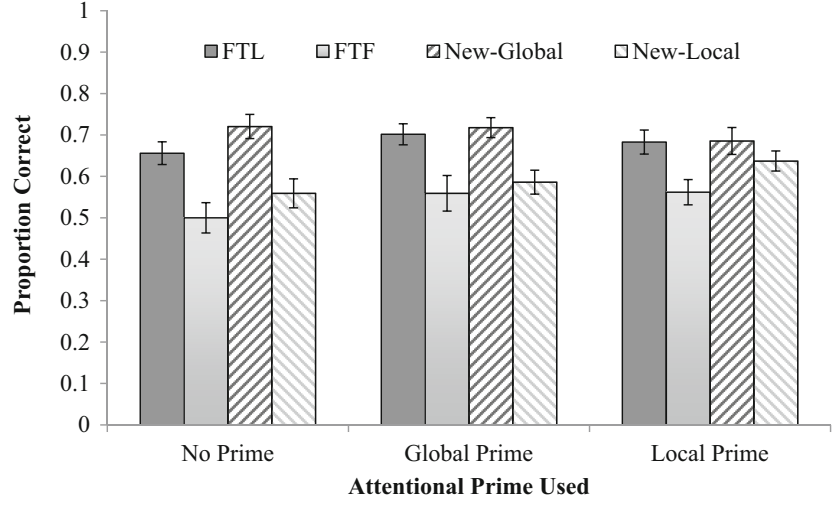

Fig. 3 Mean proportion correct in Experiment 1 for each type of change trial $(\mathrm{FTL}=$ feature-to-level; FTF $=$ feature-to-feature). Accuracy is grouped by the type of prime. Error bars represent the standard error of the mean

affected by attention, feature-to-level and new-global trials were compared (the dark gray solid and patterned bars in Fig. 3) with a 2 (trial type) $\times 3$ (attention prime) repeated measures ANOVA. New-global performance $(M=$ $.71, S E=.03)$ did not differ from feature-to-level performance $(M=.68, S E=.02), F(1,30)=1.582, p=.218$, $\eta_{\mathrm{p}}{ }^{2}=.05$. In addition, the attention prime did not affect performance, $F(2,60)=.767, p=.469, \eta_{\mathrm{p}}{ }^{2}=.03$, and there was no significant interaction between trial type and attention prime, $F(2,60)=1.076, p=.347, \eta_{\mathrm{p}}{ }^{2}=.04$.

Feature-to-feature binding To determine if individual features of hierarchical objects are bound to each other in VWM and the effect of attention on binding, performance on the feature-to-feature trials was compared with new-local performance with a 2 (trial type) $\times 3$ (attention prime) repeated measures ANOVA (the light gray solid and patterned bars in Fig. 3). New-local performance $(M=.59, S E=.03)$ was higher than feature-to-feature performance $(M=.54, S E=$ $.04), F(1,30)=6.135, p=.019, \eta_{\mathrm{p}}{ }^{2}=.17$. There was no significant effect of attention prime, $F(2,60)=2.556, p=.086$, $\eta_{\mathrm{p}}{ }^{2}=.08$, and no interaction between trial type and attention prime, $F(2,60)=.649, p=.526, \eta_{\mathrm{p}}^{2}=.02$.

False alarm rate It is possible that the false alarm rate differed as a function of the attentional prime. To determine whether the attention prime affected the false alarm rate, accuracy on the no-change trials (i.e., the proportion of no-change trials in which participants responded "present") was compared across levels of prime type. Overall false alarm rate was .34 and accuracy did not differ based on the attention prime condition, as revealed by a one-way ANOVA, $F(2,60)=.577, p=.564$, $\eta_{\mathrm{p}}{ }^{2}=.14$ (no prime $M=.34, S E=.021$; global prime $M=.33$, $S E=.024$; and local prime $M=.35, S E=.025)$.

\section{Discussion}

Experiment 1 revealed that global features are more likely to be maintained in VWM than local features and feature-to-level bindings are represented in VWM. However, there was no effect of attention prime, which suggests that feature-to-level binding may occur prior to VWM encoding. Furthermore, although features are accurately bound to their level, they are not likely to be accurately bound to each other. That is, feature-tofeature performance was lower than the more difficult feature (local features), suggesting that feature-to-feature bindings were not necessarily maintained in VWM.

Feature-to-feature bindings may be difficult to maintain in Experiment 1 because the task exceeds VWM resources (Bays, Wu, \& Husain, 2011). While the capacity of VWM is estimated to be approximately three to four simple objects, such as colored squares (Luck \& Vogel, 1997), this estimate decreases for more complex objects, such as Chinese characters (Alvarez \& Cavanagh, 2004). Three Navon figures may therefore be outside the capacity limits of most participants. According to some models of VWM, the fidelity of VWM representations decreases as VMW load increases (Bays \& Husain, 2008), which may include the memory for bindings (Bays et al., 2011). The feature-to-feature binding representations may be more sensitive to lost fidelity when traditional capacity limits are exceeded, decreasing the probability of detecting a binding change. In Experiment 2a, we manipulated VWM load to see if feature-to-feature performance would improve when there were fewer objects to encode.

It is also possible that the low feature-to-feature performance levels found in Experiment 1 resulted from encoding failures rather than maintenance failures. That is, participants may have been unable to perceive and attend to all of the items, including the global and local features and their bindings, within the $500 \mathrm{~ms}$ encoding time. This issue was addressed in Experiment 2a, in which there were fewer objects to encode and therefore more encoding time per object, and in Experiment $2 b$, in which the presentation time of the study array was manipulated while holding the number of objects constant. If encoding time limits feature-to-feature performance, then performance should improve when there is more time to encode each object.

\section{Experiment 2a}

\section{Method}

Participants Twenty-four ( 7 males and 17 females) undergraduate psychology students from Louisiana State University, with self-reported normal or corrected-to-normal vision and a mean age of 19.66 years $(S D=1.74$ years $)$, participated in the experiment for course credit. 
Stimuli and procedure Stimuli and procedure were the same as those of Experiment 1 except for the details noted here. Experiment 2a did not include an SF prime. One, two, or three Navons were displayed for $500 \mathrm{~ms}$. Navons appeared in the same spatial locations as those outlined in Experiment 1. The resulting design was a 5 (trial type) $\times 3$ (number of Navons) repeated measures.

Participants completed three blocks of 96 experimental trials; 48 no-change, 12 new-global, 12 new-local, 12 feature-tolevel, and 12 feature-to-feature. In each block, one-third of the trials were one-Navon trials, one-third were two-Navon trials, and one-third were three-Navon trials. Throughout the experiment, there were 12 new-local, 12 new-global, 12 feature-tolevel and 12 feature-to-feature for both the two-Navon and three-Navon trials. For the one-Navon trials, feature-tofeature changes were not possible, and the number of newglobal change type was doubled (24). For this reason, the oneNavon trials were not analyzed. Trials were randomized within each block, with a break incorporated between each block.

\section{Results}

The set size effect on the GPE, feature-to-level, and feature-tofeature binding was analyzed on the relevant change trials only. Proportion correct on the change trials was used to calculate accuracy for all subsequent analyses (see Fig. 4).

Global precedence effect To determine the effect of the number of Navons on the GPE, the new-global and new-local change trials were compared across the number of Navons (two, three) with a 2 (trial type) $\times 2$ (number of Navons) repeated measures ANOVA (the patterned bars in Fig. 4). This analysis revealed a GPE, as overall performance was higher on the new-global $(M=.80, S E=.02)$ than on the

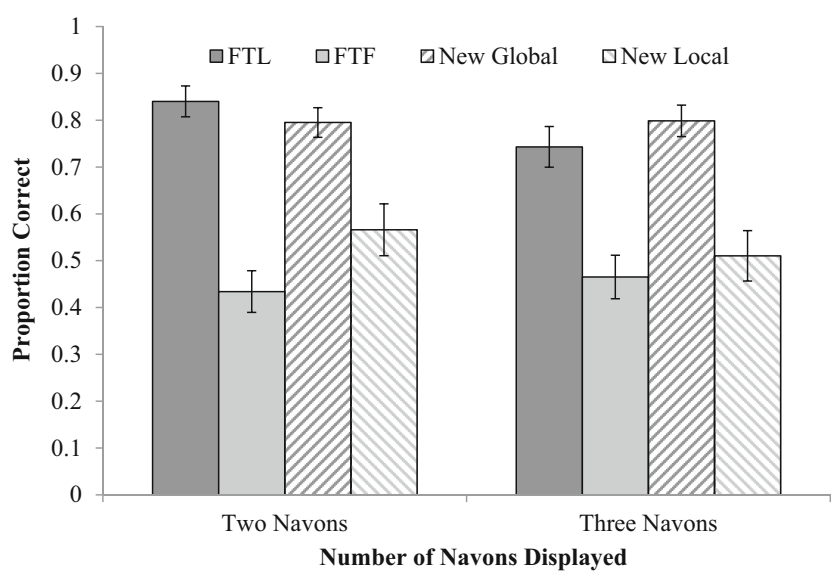

Fig. 4 Mean proportion correct in Experiment 2a for each type of change trial $(\mathrm{FTL}=$ feature-to-level; FTF=feature-to-feature). Accuracy is grouped by the number of Navons displayed. Error bars represent the standard error of the mean new-local $(M=.54, S E=.04)$ trials, $F(1,23)=17.584, p<$ $.001, \eta_{\mathrm{p}}{ }^{2}=.43$. However, there was no difference in performance between the two-Navon and three-Navon trials, $F(1$, 23) $=.744, p=.397, \eta_{\mathrm{p}}{ }^{2}=.03$. In addition, there was no interaction, $F(2,46)=1.301, p=.282, \eta_{\mathrm{p}}^{2}=.05$; therefore, the GPE was consistent across all working memory loads.

Feature-to-level binding To determine whether the number of Navons affects the feature-to-level bindings, the feature-tolevel and new-global trials were compared across the number of Navons (two, three) with a 2 (trial type) $\times 2$ (number of Navons) repeated measures ANOVA (dark gray bars in Fig. 4). There was no difference between the feature-to-level and new-global trials, $F(1,23)=.035, p=.853, \eta_{\mathrm{p}}{ }^{2}=.00$, and no main effect for the number of Navons, $F(1,23=2.221, p=$ $.150, \eta_{\mathrm{p}}^{2}=.09$; however, a significant trial type by number of Navons interaction was observed, $F(1,23)=5.235, p=.032$, $\eta_{\mathrm{p}}^{2}=.19$.

To investigate the interaction, pairwise comparisons were performed. Analysis revealed that on the feature-to-level trials, performance was higher for two Navons $(M=.84$, $S E=.03)$ than for three Navons $(M=.74, S E=.04), t(23)=$ $2.228, p=.036$; however, there was no difference in the newglobal trials across the number of Navons (two Navons $M=$ $.80, S E=.03$; three Navons $M=.80, S E=.05), t(23)=.107, p$ $=.915$. Additionally, there were no differences between the new-global and feature-to-level for either the two Navon, $t(23)$ $=1.357, p=.19$, or the three-Navon conditions $t(23)=1.484$, $p=.151$. Therefore, decreasing the number of Navons improves level binding but does not improve new-global performance.

Feature-to-feature binding To determine whether the number of Navons affects feature-to-feature bindings, performance for feature-to-feature and new-local changes was compared across the number of Navons (two, three) with a 2 (trial type) $\times 2$ (number of Navons) repeated measures ANOVA (light gray bars in Fig. 4). Feature-to-feature change detection was lower $(M=.45, S E=.04)$ than new-local $(M=.59, S E=.04)$ performance, $F(1,23)=5.507, p=.028, \eta_{\mathrm{p}}^{2}=.19$. There was no effect for the number of Navons, $F(1,23)=.123, p=.729$, $\eta_{\mathrm{p}}^{2}=.01$, and no significant interaction, $F(1,23)=2.387, p=$ $.136, \eta_{\mathrm{p}}{ }^{2}=.09$. Therefore, decreasing the number of Navons did not improve maintenance of feature-to-level bindings.

False alarm rate To determine whether the number of Navons affected the false alarm rate, no-change trials were analyzed with a paired samples $t$ test. This $t$ test was significant, $t(23)=2.77, p<.01$, as the false alarm rate on the twoNavon trials $(M=.30, S E=.025)$ was lower than false alarm rate on the three Navon trials $(M=.38, S E=.036)$. 


\section{Discussion}

Consistent with the results from Experiment 1, a GPE was present, and features were bound to their levels but not to each other. Decreasing the number of Navons improved performance in some situations (feature-to-feature change detection was higher and false alarm rate was lower with two Navons than with three Navons), but the basic patterns of the results was the same as those found in Experiment 1. Importantly, feature-to-feature bindings were not well maintained, even when there were only two objects to encode into VWM, suggesting that the low rate of feature-to-feature binding found in Experiment 1 was not due to high VWM load. These results also suggest that the encoding time per Navon is not the reason for low feature-to-feature performance because even when the same encoding time could be devoted to two Navons rather than three (as in Experiment 1), feature-to-feature performance was still poor. However, to test this possibility directly, presentation time was manipulated in Experiment $2 \mathrm{~b}$. Specifically, presentation time was increased to determine if additional encoding time improves feature-to-feature performance.

\section{Experiment 2b}

\section{Method}

Participants Twenty-five ( 5 males and 20 females) undergraduate psychology students from Louisiana State University, with self-reported normal or corrected-to-normal vision and a mean age of 20.25 years $(S D=1.22$ years) participated in the experiment for course credit.

Stimuli and procedure Stimuli and procedure were the same as those of Experiment 1 except for the details noted here. Experiment $2 \mathrm{~b}$ did not include a SF prime. Three Navons were presented for either $500 \mathrm{~ms}, 1,000 \mathrm{~ms}$, or 1,500 ms, blocked separately, with block order randomized across participants. The design was a 5 (trial type) $\times 3$ (display duration) repeated measures. There were 96 randomized trials within three blocks (one block for each encoding duration), with 48 nochange, 12 new-global, 12 new-local, 12 feature-to-level, and 12 feature-to-feature trials in each block.

\section{Results}

The effect of encoding time on the GPE, feature-to-level bindings, and feature-to-feature bindings was examined by analyzing performance on only the trials with changes (see Fig. 5).

Global precedence effect The effect of encoding time on the GPE was determined by comparing the new-global and new-

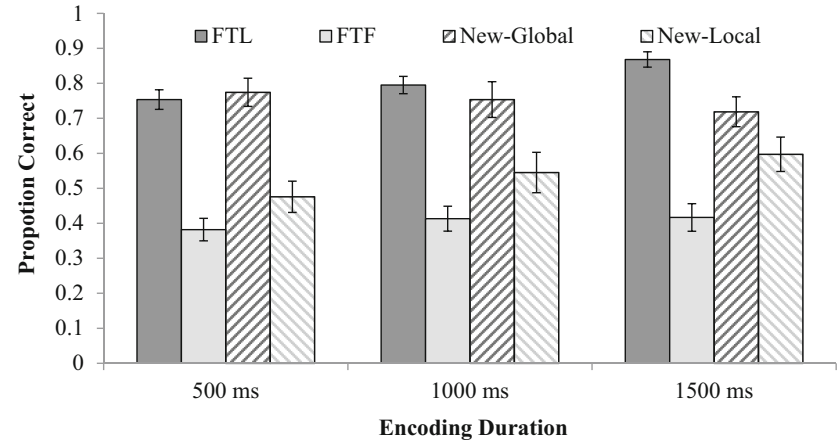

Fig. 5 Mean proportion correct in Experiment $2 b$ for each type of trial with a change $(\mathrm{FTL}=$ feature-to-level; $\mathrm{FTF}=$ feature-to-feature). Accuracy is grouped by the encoding duration. Error bars represent the standard error of the mean

local trials across encoding duration $(500,100,1,500 \mathrm{~ms})$ with a 2 (trial type) $\times 3$ (display duration) repeated measures ANOVA. There was a marginal effect of trial type, $F(1,24)$ $=3.070, p=.056, \eta_{\mathrm{p}}{ }^{2}=.11$, and no effect of display duration, $F(1,24)=2.616, p=.119, \eta_{\mathrm{p}}{ }^{2}=.10$. However, there was a significant interaction, $F(2,48)=8.910, p=.001, \eta_{\mathrm{p}}{ }^{2}=.27$. This is because longer display times eliminated the GPE: there was a significant GPE for the 500, $t(24)=4.114, p<.001$, and $1,000 \mathrm{~ms}, t(24)=2.353, p=.027$, display durations, but not for the $1,500 \mathrm{~ms}$ duration $t(24)=1.602, p=.122$.

Feature-to-level binding To examine whether encoding time affected feature-to-level binding, feature-to-level trials were compared to new-global trials across all encoding durations $(500,1,000,1,500 \mathrm{~ms})$ with a 2 (trial type) $\times 3$ (display duration) repeated measures ANOVA. There was no effects of trial type, $F(2,48)=1.731, p=.201, \eta_{\mathrm{p}}{ }^{2}=.08$, or display duration, $F(2,48)=.469, p=.629, \eta_{\mathrm{p}}{ }^{2}=.02$, but there was a significant interaction, $F(2,48)=5.146, p=.008, \eta_{\mathrm{p}}{ }^{2}=.18$. Specifically, feature-to-level performance was higher than new-global at 1 , $500 \mathrm{~ms}, t(24)=2.986, p=.00$, but there was no difference between these conditions at $500 \mathrm{~ms}, t(24)=.539, p=.595$ or at $1,000 \operatorname{mst}(24)=.733, p=.471$. Therefore, the increased encoding time improved feature-to-level binding.

Feature-to-feature binding To assess the effect of display duration on feature-to-feature bindings, new-local trials were compared to feature-to-feature binding trials with a 2 (trial type) $\times 3$ (display duration) repeated measures ANOVA. Feature-to-feature performance $(M=.40, \mathrm{SE}=.04)$ was lower than new-local performance $(M=.54, S E=.05), F(2,48)=$ $7.924, p=.010, \eta_{\mathrm{p}}{ }^{2}=.29$. However, there was no effect of display duration, $F(2,48)=2.312, p=.110, \eta_{\mathrm{p}}{ }^{2}=.09$, and no significant interaction, $F(2,48)=1.817, p=.174, \eta_{\mathrm{p}}{ }^{2}=.07$. Therefore, feature-to-feature performance was lower than new-local performance regardless of the display duration. 
False alarm rate To determine whether display duration affected the false alarm rate, a one-way repeated measures ANOVA was conducted on false alarm rate on the nochange trials. Longer encoding times tended to reduce the rate of false alarms, $F(2,48)=5.357, p=.008, \eta_{\mathrm{p}}{ }^{2}=.18$. Specifically, as revealed by a post hoc LSD test, performance was lower with the $500 \mathrm{~ms}$ encoding duration $(M=.35, S E=$ $.03)$ than with the $1,000 \mathrm{~ms}(M=.29, S E=.03), p=.019$, or 1 , $500 \mathrm{~ms}(M=.27, S E=.03), p=.003$. However, no significant differences were observed for no-change trials between $1000 \mathrm{~ms}$ and $1500 \mathrm{~ms}$ conditions, $p=.387$.

\section{Discussion}

Experiment $2 \mathrm{~b}$ expanded upon Experiment $2 \mathrm{a}$ by directly manipulating encoding duration. Longer encoding times did affect the GPE, which decreased as encoding duration increased, likely because local features were more likely to be encoded as encoding time increased. Furthermore, performance for the feature-to-level bindings improved as the encoding duration increased, with feature-to-level performance eventually exceeding new-global accuracy at the 1 , $500 \mathrm{~ms}$ display duration. This is probably because the longer encoding times increased the amount of local information available in VWM (as evidenced by the lack of a GPE for the 1,500 ms condition). Furthermore, the concurrent improvement of new-local and feature-to-level performance suggests that features are automatically bound to their hierarchical in VWM. However, despite this increase in new-local information at longer encoding times, there was no benefit for feature-to-feature bindings with increased encoding duration. This suggests that features are not automatically bound to each other, even with extended encoding time (Cowan, Blume, \& Saults, 2013).

\section{Experiment 3}

It is possible that the effects observed in the preceding experiments are partially due to verbal encoding of the letters and may not represent how hierarchical objects are stored in VWM. Therefore, Experiment 3 was conducted to see if the GPE, feature-to-level, and the feature-to-feature binding effects would still be present when articulatory suppression minimized the ability to verbally encode the letters.

\section{Method}

Participants Twenty-six (6 males and 20 females) undergraduate psychology students from Louisiana State University, with self-reported normal or corrected-to-normal vision and a mean age of 20.12 years ( $S D=1.31$ years), participated in this experiment for course credit.
Stimuli and procedure Stimuli and procedure were the same as those of Experiment $2 \mathrm{~b}$ except for the details noted here. Experiment 3 included an articulatory suppression task. Prior to the start of each trial, two different randomly selected single-digit, single-syllable numbers (i.e., 1, 2, 3, 4, $5,6,8$, and 9) were presented to the participant. The participant was tasked with verbally repeating the numbers from the onset of the study array until the response screen was presented. Using the built-in computer microphone, articulation was monitored by using the PsychSound function in the Psychophysics Toolbox (Brainard, 1997; Pelli, 1997). The audio was recorded at $44.1 \mathrm{kHz}$, and audio information that was at least $5 \%$ of the maximum allowable recording volume was determined to be an articulation. This criterion was chosen as it reflected a recording above the peak amplitude of each waveform for each recorded sample. After a sample above threshold was found, $50 \mathrm{~ms}$ was advanced in the audio recording before another volume assessment was created. Based on these recording and assessment criteria, a participant needed to complete at minimum four articulations during the course of a trial in order for a trial to be considered valid ( $M=6.95, S E=.03$ articulations per trial). If the participant had not completed the articulation minimum, or not spoken loudly enough to be considered a valid recording, a warning screen would be displayed following the trial, alerting the participant to speak louder and more quickly. Trials for which participants failed to meet the articulation criteria were flagged and removed from subsequent analyses. The mean number of trials removed from across all participants was 12.3 trials $(S E=2.51)$. To ensure that a single trial type was not more likely to be removed, a one-way repeated measures ANOVA was conducted on the overall proportion of trials removed for the five trial types (no-change: $M=.05, S E=.01$; feature-to-level: $M=.05, S E$ $=.01$; feature-to-feature: $M=.04, S E=.01$; new-global: $M=$ $.05, S E=.01$; and new-local: $M=.04, S E=.01$ ). Analysis revealed no significant differences in the overall proportion of trials removed for failing to perform the articulatory suppression task, $F(4,100)=.607, p=.658, \eta_{\mathrm{p}}^{2}=.02$.

Following the presentation of the numbers, the change detection task would begin. This was identical to Experiment $2 b$, except that only the 1,000 ms display time was utilized. This resulted in a one-way repeated measures design with 5 (trial type) levels. There were four blocks of 64 randomized trials each, with 32 no-change, eight new-global, eight new-local, eight feature-to-level, and eight feature-to-feature trials in each block.

\section{Results}

The effect of verbal coding on the GPE, feature-to-level bindings, and feature-to-feature bindings was examined by analyzing performance on the trials with changes (see Fig. 6). 


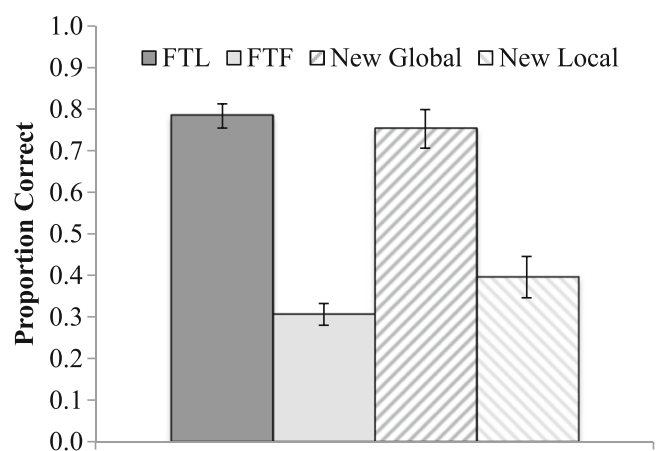

Fig. 6 Proportion correct for Experiment 3 (FTL=feature-to-level; $\mathrm{FTF}=$ feature-to-feature). Error bars represent standard errors of the mean

Global precedence effect A GPE was present, as a paired samples $t$ test revealed that new-global performance $(M=$ $.75, S E=.05)$ was higher than new-local performance $(M=$ $.40, S E=.05), t(25)=4.04, p<.001$.

Feature-to-level binding Feature-to-level performance $(M=$ $.78, S E=.03)$ did not differ from new-global $(M=.75, S E=$ $.05)$, as revealed by a paired samples $t$ test, $t(25)=.67, p=$ .511 .

Feature-to-feature binding Feature-to-feature performance $(M=.31, S E=.03)$ was lower than new-local performance $(M=.40, S E=.05)$, as revealed by a paired samples $t$ test, $t(25)=2.10, p=.046$.

False alarm rate No-change performance was similar to previous experiments $(M=.73, S E=.02)$; the false alarm rate for the no-change trials was .27 . As a direct comparison to evaluate the effect of the articulatory load on the false alarm rate, an independent-samples $t$ test was conducted between the nochange trials of Experiment 3 and the 1,000 ms no-change trials of Experiment $2 \mathrm{~b}(M=.29, S E=.03)$. Analysis revealed no significant difference, $t(49)=.483, p=.631$.

\section{Discussion}

With an articulatory suppression task, the results of Experiment 3 replicated those found in the previous experiments. That is, a global precedence effect was present in VWM, and feature-to-level bindings were well maintained while feature-to-feature bindings were not. Therefore, verbal encoding cannot account for the GPE in VWM or the poor feature-to-feature binding memory.

\section{General discussion}

The results of the current study demonstrate that global features are more easily stored in VWM, both global and local levels are bound to their hierarchical levels, but the features of an object are not necessarily bound to each other. This was true regardless of set size, encoding time, and verbal suppression, with the exception that longer encoding times improved memory for local features. Together, these results suggest that feature-to-level binding may be a relatively automatic process for items encoded into VWM, while feature-to-feature binding is not.

\section{The global precedence effect in VWM}

Across four experiments, new-global performance was higher than new-local performance (with the exception of long encoding duration), demonstrating a robust GPE in VWM. Interestingly, the GPE was not eliminated by either directing attention to the local level (see Experiment 1) or by decreasing the number of items to encode (Experiment $2 \mathrm{a}$ ) or by adding a verbal suppression (Experiment 3). However, increasing encoding time did improve new-local performance and thereby diminish the GPE (see Experiment 2b). This suggests that with sufficient encoding time, local features can be encoded as well as global features. However, in Experiment 2a, decreasing the total number of objects to one Navon did not affect the GPE in VWM, even though this resulted in the same encoding time-per-object as the 1,500 ms condition of Experiment $2 \mathrm{~b}$. Therefore, overall encoding time, rather than time per object, appears to be critical in improving new-local performance. Local features take more time to encode into VWM, and this increased encoding time is not dependent on the focus of attention or the number of items in the display.

\section{Feature-to-level binding for hierarchical objects}

Across several manipulations (biasing attention, decreasing set size, increasing encoding time, and minimizing verbal encoding) new-global and feature-to-level change detection performance remained consistently high. This suggests that encoding global information and feature-to-level bindings may be obligatory, much like encoding location information appears to be (Tsal \& Lavie, 1988; van Lamsweerde \& Beck, 2011). In Experiment 2b, at the longest encoding time, feature-to-level performance was higher than new-global performance, and this was accompanied by an increase in newlocal performance. This suggests that as long as there was enough time to encode the local information, the local feature-to-level binding information was also encoded.

The low feature-to-feature binding performance suggests that it is unlikely that global and local features were stored together as a bound object in VWM, a strategy that has been shown to improve change detection performance (Luck \& Vogel, 1997). However, although the global and local features were not bound together to maximize capacity, they also did not appear to compete for VWM resources: improving new- 
local performance did not result in a decrease in new global performance. It is possible that the global "letter" is actually encoded in VWM as a spatial configuration (Simons, 1996), similar to location information, and therefore does not compete for VWM resources with local features of an object.

\section{Feature-to-feature binding for hierarchical objects}

Previous feature-to-feature binding research has primarily focused on whether features within a level are bound to each other (e.g., colored shapes: Fougnie \& Marois, 2009; Johnson et al., 2008; Wheeler \& Treisman, 2002). For nonhierarchical objects, certain attention manipulations (e.g., multiple object tracking) and testing conditions (e.g., a whole array test) can disrupt feature-to-feature binding memory more than feature memory (e.g., detect a new shape), suggesting that binding memory may be more attention demanding, fragile, or vulnerable to disruption than feature memory (Allen et al., 2006; Alvarez \& Cavanaugh, 2008; Baddeley, Allen, \& Hitch, 2011; van Lamsweerde \& Beck, 2012; Wheeler \& Treisman, 2002). However, in the absence of these attention and/or testing manipulations, within-level feature-to-feature bindings are often detected as well as changes to the more difficult feature, which suggests that the bindings are encoded into VWM (Allen et al., 2006; Brown \& Brockmole, 2010; Johnson et al., 2008; van Lamsweerde \& Beck, 2011; Wheeler \& Treisman, 2002; Woodman \& Vogel, 2008, but see van Lamsweerde, Beck, \& Elliott, 2015). In contrast, in the current study, feature-to-feature binding changes were more difficult to detect than new-local (difficult feature) changes, suggesting that feature-to-feature bindings may be specifically difficult to maintain across hierarchical levels. Although this study does not directly compare within-level feature-to-feature bindings (e.g., Wheeler \& Treisman, 2002) with across-level feature-tofeature bindings, the data here suggest that across-level feature-to-feature bindings may be more difficult to maintain in VWM. Sustained attention may therefore play a more important role in maintaining an integrated representation for a hierarchical object than a single-level object in VWM, although this requires additional research to confirm.

Furthermore, while the data here suggest that features may be bound to their levels automatically, this was not the case with feature-to-feature binding, which suggests that binding memory may differ depending on the information being bound. Similarly, color and orientation of an object have been reported to fail independently in VWM, while the height and width of an object were more likely to be remembered together (Fougnie \& Alvarez, 2011). This suggests that the interdependence of two features can determine how likely they are to be bound together in VWM. Likewise, a feature and its level may be more integral than features at two different levels, resulting in poorer feature-to-feature binding in VWM.
It is worth noting that although feature-to-feature performance was always lower than new-local performance, it was not the case that the hierarchical features of an object were never bound together in VWM. Across the 10 conditions used in the four experiments, the average size of the difference between the new-local and the feature-to-feature trials was approximately $9 \%$. Furthermore, across all the experiments, average hit rate for feature-to-feature changes $(M=.43, S E=$ $.02)$ was statistically greater than the average rate of false alarms (i.e., saying the test item was not present in the study array on a no-change trial) across the experiments $(M=.31$, $S E=.01), t(105)=8.103, p<.001$. This indicates that features were accurately bound together across hierarchical levels for some of the items in VWM.

\section{Encoding and response biases}

It is important to note that although we interpret the current results to reflect VWM storage, it is possible that performance is also affected by biases at the encoding or the decision/response stage of processing. In the current studies, the task design was chosen in order to encourage complete and bound representations of the objects. Specifically, the change types were randomly intermixed so that at encoding participants did not know which change type could occur. Regardless, it is possible that participants adopted a strategy of only encoding one feature and ignoring the other feature. However, while this could potentially explain the GPE, it could not explain the poor feature-tofeature performance, because the feature-to-feature binding could still be encoded as well as the feature that was encoded second (local features).

It is also possible that there was a response bias or different criterion for detecting different change types. Specifically, in the current study, the trial types were randomly intermixed, which means participants were encouraged to encode all of the features and their bindings; however, as a result, the false alarm rates cannot be determined for each change type. When false alarms were made on the no-change trials, there was no way to know what type of change the participants thought they had detected. Therefore, it is possible that all features and bindings were stored in VWM in a bound and complete representation but that only some of these features and bindings were accessible when making a decision about the change. This would indicate that VWM representations are not necessarily accessed as bound representations during the change detection process, and that each feature is compared individually, with each feature having a potentially different response criterion. Although it seems unlikely that each change type would have a different response criterion, the current study cannot rule out this possibility. 


\section{Conclusion}

The current study suggests that global features are encoded more readily than local features, and that although the hierarchical level is bound to the features, the features are not necessarily bound to each other. This suggests that these two types of bindings may be represented differently in VWM. The exact mechanism for these discrepancies requires further exploration. Global features may be remembered more like a spatial configuration (Simons, 1996) than like a feature, resulting in greater global performance and a lack of competition between global and local features. Features and their levels may also be perceived as intrinsic properties, whereas two features at different hierarchical levels may not. This could result in automatic feature-to-level, but not feature-tofeature bindings. However, these possibilities require additional research.

\section{References}

Allen, R. J., Baddeley, A. D., \& Hitch, G. J. (2006). Is the binding of visual features in working memory resource-demanding? Journal of Experimental Psychology: General, 135(2), 298-313.

Allen, R. J., Hitch, G. J., Mate, J., \& Baddeley, A. D. (2012). Feature binding and attention in working memory: A resolution of previous contradictory findings. The Quarterly Journal of Experimental Psychology, 65, 2369-2383. doi:10.1080/17470218.2012.687384

Alvarez, G. A., \& Cavanagh, P. (2004). The capacity of visual short-term memory is set both by visual information load and by number of objects. Psychological Science, 15(2), 106-111. doi:10.1111/j.09637214.2004.01502006.x

Alvarez, G. A., \& Cavanagh, P. (2008). Visual short-term memory operates more efficiently on bondary features than on surface features. Perception \& Psychophysics, 70(2), 346-364. doi:10.3758/ PP.70.2.346

Austen, E., \& Enns, J.T. (2000). Change detection: Paying attention to detail. Psyche: An Interdisciplinary Journal of Research on Consciousness, 6(11).

Baddeley, A. D., Allen, R. J., \& Hitch, G. J. (2011). Binding in visual working memory: The role of the episodic buffer. Neuropsychologia, 49, 1393-1400. doi:10.1016/j. neuropsychologia.2010.12.042

Bays, P. M., \& Husain, M. (2008). Dynamic shifts of limited working memory resources in human vision. Science, 321(5890), 851-854. doi:10.1126/science. 1158023

Bays, P. M., Wu, E. Y., \& Husain, M. (2011). Storage and binding of object features in visual working memory. Neuropsychologia, 49(6), 1622-1631. doi:10.1016/j.neuropsychologia.2010.12.023

Boer, C. L., \& Keuss, P. J. G. (1982). Global precedence as a postperceptual effect: An analysis of speedaccuracy trade-off functions. Perception \& Psychophysics, 31(4), 358-366. doi:10.3758/ BF03202660

Brainard, D. H. (1997). The psychophysics toolbox. Spatial Vision, 10, 433-436.

Brown, A. L., \& Brockmole, J. R. (2010). The role of attention in binding visual features in working memory: Evidence from cognitive ageing. The Quarterly Journal of Experimental Psychology, 63(10), 2067-2079.
Cowan, N., Blume, C. L., \& Saults, J. S. (2013). Attention to attributes and objects in working memory. Journal of Experimental Psychology: Learning, Memory, and Cognition, 39, 731-747.

Delvenne, J.-F., Cleeremans, A., \& Laloyaux, C. (2010). Feature bindings are maintained in visual short-term memory without sustained focused attention. Experimental Psychology, 57, 108-116.

Flevaris, A. V., Bentin, S., \& Robertson, L. C. (2010). Local or global?: Attentional selection of spatial frequencies binds shapes to hierarchical levels. Psychological Science, 21(3), 424-431.

Fougnie, D., \& Alvarez, G. A. (2011). Object features fail independently in visual working memory: Evidence for a probabilistic feature-store model. Journal of Vision, 11, 1-12.

Fougnie, D., \& Marois, R. (2009). Attentive tracking disrupts feature binding in visual working memory. Visual Cognition, 17, 48-66. doi:10.1080/13506280802281337

Gajewski, D. A., \& Brockmole, J. R. (2006). Feature bindings endure without attention: Evidence from an explicit recall task. Psychonomic Bulletin and Review, 13, 581-587.

Hübner, R., \& Volberg, G. (2005). The integration of object levels and their content: A theory of global/local processing and related hemispheric differences. Journal of Experimental Psychology: Human Perception and Performance, 31, 520-541.

Johnson, S. J., Hollingworth, A., \& Luck, S. J. (2008). The role of attention in the maintenance of feature bindings in visual short-term memory. Journal of Experimental Psychology: Human Perception and Performance, 34(1), 41-55.

Lamb, M. R., \& Yund, E. W. (1993). The role of spatial frequency in the processing of hierarchically organized stimuli. Perception \& Psychophysics, 54, 773-784.

Lamb, M. R., \& Yund, E. W. (1996). Spatial frequency and interference between global and local levels of structure. Visual Cognition, 3, 193-219.

Luck, S. J., \& Vogel, E. K. (1997). The capacity of visual working memory for features and conjuctions. Nature, (390), 279-281. doi:10. $1038 / 36846$

Miller, J. (1981). Global precedence in attention and decision. Journal of Experimental Psychology: Human Perception and Performance, 7(6), 1161-1174.

Morey, C. C., \& Bieler, M. (2013). Visual short-term memory always requires general attention. Psychonomic Bulletin \& Review, 20, 163-170. doi:10.3758/s13423-012-0313-z

Navon, D. (1977). Forest before the tress: The precedence of global features in visual perception. Cognitive Psychology, 9, 353-393.

Pelli, D. G. (1997). The videotoolbox software for visual psychophysics: Transforming numbers into movies. Spatial Vision, 10, 437-442.

Rumiati, R., Nicoletti, R., \& Job, R. (1989). Processing of global and local information in memory. Quarterly Journal of Experimental Psychology Section A: Human Experimental Psychology, 41(1), 167-181.

Simons, D. J. (1996). In sight, out of mind: When object representations fail. Psychological Science, 7, 301-305.

Tsal, Y., \& Lavie, N. (1988). Attending to color and shape: The special role of location in selective visual processing. Perception \& Psychophysics, 44(1), 15-21. doi:10.3758/BF03207469

Treisman, A. M., \& Gelade, G. (1980). A feature-integration theory of attention. Cognitive Psychology, 12, 97-136.

van Lamsweerde, A. E., \& Beck, M. R. (2011). The change probability effect: Incidental learning, adaptability, and shared visual working memory resources. Consciousness and Cognition, 20, 1676-1689.

van Lamsweerde, A. E., \& Beck, M. R. (2012). Attention shifts or volatile representations: What causes binding deficits in visual working memory? Visual Cognition, 20(7), 771-792. 
van Lamsweerde, A. E., Beck, M. R., \& Elliott, E. M. (2015). Retrieval from long-term memory reduces working memory representations for visual features and their bindings. Memory \& Cognition, 43(2), 237-246. doi:10.3758/s13421-014-0468-0

Wheeler, M. E., \& Treisman, A. M. (2002). Binding in short-term visual memory. Journal of Experimental Psychology: General, $131,48-64$.
Woodman, G. F., \& Vogel, E. (2008). Selective storage and maintenance of an object's features in visual working memory. Psychonomic Bulletin \& Review, 15(1), 223-229.

Yeh, Y.-Y., Yang, C.-T., \& Chiu, Y.-C. (2005). Binding or prioritization: The role of selective attention in visual short-term memory. Visual Cognition, 12, 759-799. 